\title{
Integrated Capacitance Sensing for Miniature Artificial Muscle Actuators
}

\author{
Julie Legrand, Bart Loenders, Alexander Vos, Laurent Schoevaerdts and Emmanuel Vander Poorten
}

\begin{abstract}
The use of McKibben pneumatic artificial muscles in surgical instruments is increasing due to their light weight, miniaturization potential, compliance and large actuation force. However, precise position and force control is still challenging due to the intrinsic muscle hysteresis. The present work proposes an easy-to-make integrated capacitance sensor for miniature artificial muscles by replacing two strands of the muscle braid with two conductive wires. By measuring the capacitance at high excitation frequency between both wires, the length of the muscle can be deduced. This new integrated sensor still allows the miniaturization of McKibben muscles. The miniature muscle with integrated capacitance sensing only loses $3 \%$ of its contraction length with regard to an original McKibben muscle. The sensor can predict the muscle length with a $0.31 \mathrm{~mm}$ precision over $\mathbf{8 0} \%$ of the muscle total achievable contraction range in average.
\end{abstract}

Index Terms-McKibben actuator, capacitance, intelligent structures, robot sensing systems.

\section{INTRODUCTION}

M CKIBBEN pneumatic artificial muscles are increasingly being used as actuators in surgical instruments [1-5]. These artificial muscles consist of an internal bladder, surrounded by a non-extensible braided mesh. When the internal bladder is pressurized, the bladder inflates. Due to the non-extensibility of the braided mesh threads, the actuator shortens as the volume increase [6]. Their simple design, light weight, miniaturization potential, large power density and operational bandwidth make McKibben muscles particularly suitable to be implemented in surgical instruments dealing with delicate manipulations such as sinus, skull base or fetal surgery [2]. McKibben muscles also offer large actuation force and strokes [2]. However, the hysteresis present during the muscle inflation or deflation, makes the control of McKibben muscles challenging. This hysteresis originates from the intrinsic hysteresis of the bladder, friction between the braided mesh and the bladder, and friction between the mesh strands themselves [7].

In order to improve the muscle positioning precision, some control techniques have been introduced in the literature. Xie et al. [8] proposed a modified generalized Prandtl-Ishlinskii model for hysteresis compensation of pneumatic artificial muscles. This model is used in feed forward, allowing an open-loop control of the muscle. Capace et al. [9] introduced a multistate friction model in order to compensate for the muscle hysteresis. These methods do not require extra

J. Legrand, B. Loenders, A. Vos, L. Schoevaerdts and E. Vander Poorten are with the Department of Mechanical Engineering Technology, Katholieke Universiteit Leuven, Heverlee B-3001, Belgium.

Corresponding author (Julie Legrand): julie.legrand@kuleuven.be sensors and thus provides a compact system. However, the accuracy and reliability of such approaches are limited. In robotic surgery, more specifically, a direct measurement of the actuator state is desired in order to increase safety. Researchers have investigated small and compact sensors dedicated for McKibben muscles.

Akagi et al. [10] developed a sensor to assess the inner diameter of a McKibben muscle. The system, composed out of two photo reflectors, is placed inside a muscle to evaluate the distance between both sensors and the inner wall, therefore giving information about the inner diameter of the muscle during actuation. Another system, using an electrically conductive rubber cylinder inside a muscle was developed by Wakimoto et al. [11]. In the latter system, the muscle bladder and braided mesh are fixed at both ends of the sensitive rubber. When actuated, the rubber is compressed, which changes its electrical resistance. Hence, measurement of the electrical resistance directly correlates to the muscle contraction. Although both systems show good results, they are quite complex to build and their miniaturization is limited.

Some prototypes were designed with a sensor placed outside the McKibben muscle. For example, a coil wrapped around the muscle fibers establishes a link between the coil inductance and the muscle contraction [12]. Kuriyama et al. and Yamamoto et al. placed respectively a flexible ring [13] or film [14] made out of an electro-conductive rubber around a muscle. The rubber resistance then correlates to the muscle diameter or shrinkage during actuation. Another concept was introduced by King and Park et al., who made use of a 3D helical soft sensor positioned around the muscle [15], [16]. This soft sensor consists out of a cured silicone layer on which a liquid metal helical pattern is printed. Again, the soft sensor electrical resistor varies as the muscle length changes. Another example, introduced by Hamamoto et al., used a flexible potentiometer placed on the braided mesh of the muscle [17]. Overall, these approaches add volume outside the muscle, complicating miniaturization.

Two solutions were proposed that integrate a sensor directly in a muscle without changing the inner or outer dimensions. Goulbourne et al. proposed a muscle bladder made out of a dielectric elastomer [18]. The capacitance of the bladder allows deducing the muscle contraction. However, no experimental data was reported for that sensor. Felt et al. proposed a braided mesh made from insulated wires connected in series [19], [20]. The inductance of this braided mesh varied upon muscle contraction and elongation. While this approach works well and shows good linearity, the 
muscle with integrated smart braid developed by Felt et al. is rather large $(9.5 \mathrm{~mm}$ outer diameter and $300 \mathrm{~mm}$ length). Since the inductance is proportional to the squared number of windings and to the squared muscle diameter, it becomes challenging to miniaturize such a sensor, considering that the targeted muscle dimensions within the scope of this work are $\leq 3.5 \mathrm{~mm}$ diameter and $\leq 110 \mathrm{~mm}$ length. The measured amplitude for this measuring approach would therefore drop significantly for the aimed dimensions. Moreover, manufacturing such conductive braid requires expensive and dedicated hardware. This study proposes a new easy-to-make sensor implementable in miniature McKibben muscles. In contrast to the governing state-of-the-art, a capacitive approach is developed. This new integrated capacitance sensing system still allows miniaturization of McKibben muscles, and allows to predict the muscle length.

The paper is structured as follows. First, a brief summary of the actuation principle of a McKibben muscle is given. The sensor concept is then presented and the theoretical sensor model is established in section II. In order to compare the results with the derived model, experimental tests are performed in section III. A full characterization of the capacitive sensor is then performed in section IV, and the results are subsequently discussed. Finally, the section V concludes the study and proposes future research tracks.

\section{SENSOR CONCEPT}

\section{A. McKibben muscle actuation principle}

A McKibben pneumatic artificial muscle is composed of an internal bladder reinforced by a non-extensible braided mesh (Fig. 1a). The internal bladder is connected to an air supply. By increasing the air pressure $(P)$, the bladder tends to expand radially. Since the braid length is constant, the braid angle $(\theta)$ increases and the actuator contracts (Fig. 1b). This property is used to generate the muscle actuation stroke [2]. The contraction range of McKibben muscle actuators can range between 20 to $35 \%$ of its initial length depending on the braid angle $\theta$ [21], [22].

\section{B. Sensor working principle}

The capacitive sensor is integrated during the fabrication process of a standard McKibben muscle. Its integration is straightforward. One strand of the muscle braided mesh is removed and replaced by one thin conductive wire. A second conductive wire is placed between two muscle strands at equal distance of the first conductive wire all along the muscle length. The conductive wires are placed in a similar fashion as the original strands in order to maximally preserve the contractile properties of the original muscle. Both conductive strands therefore form two helical parallel wires integrated in the original braided mesh. Figure 2 illustrates the working principle of the integrated capacitive sensor. The conductive wires are powered at one muscle end with an electric potential difference. They are isolated from each other at the other muscle end (open-circuit) to create a capacitive effect between both wires. When the muscle contracts, its diameter
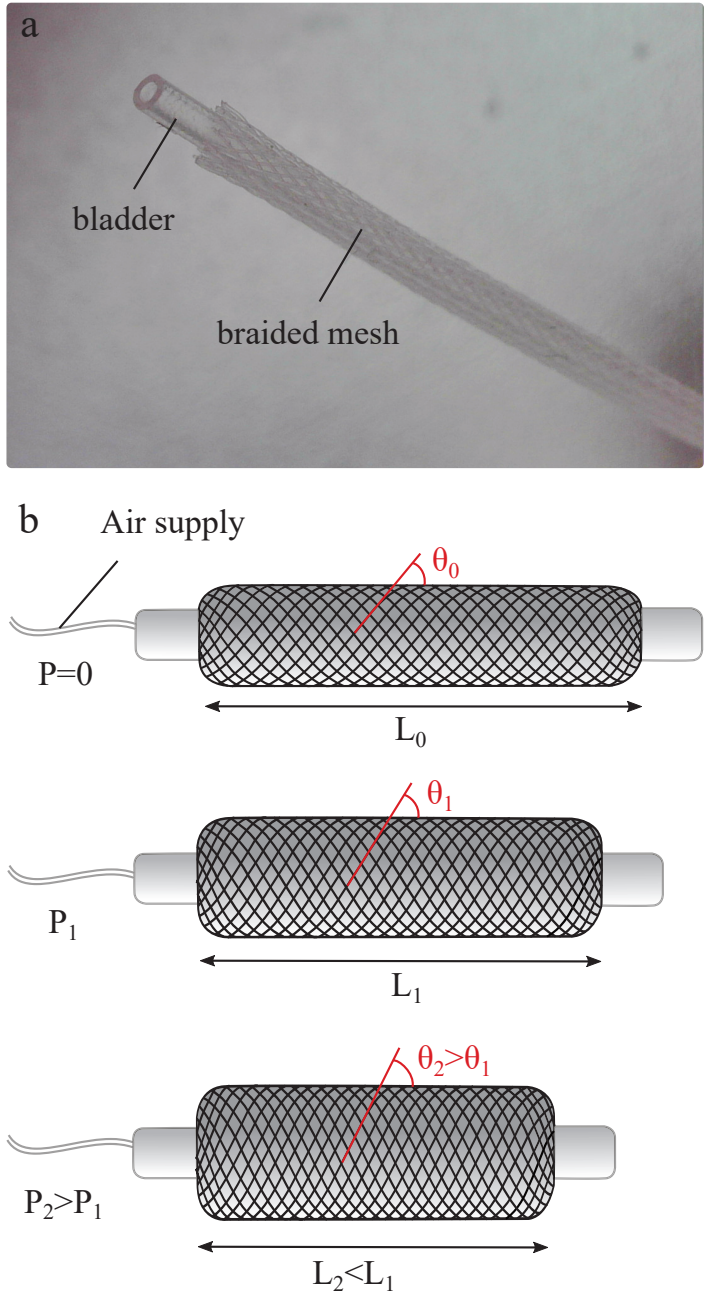

Fig. 1. Composition and actuation principle of a McKibben muscle; a) McKibben muscle components. b) muscle actuation principle: $P$, the input pressure; $L$, the actuator length; $\theta$, the braid angle. When the input pressure $P$ increases, the bladder expands radially. Since the braid length is constant, the braid angle $\theta$ increases, and the actuator length $L$ decreases.

increases and the distance between the meshed braid strands increases. Both conductive wires therefore move away from each other, leading to a decrease of the capacitance between the conductive wires.

\section{Sensor theoretical model}

The following hypotheses were made in order to model the capacitive effect of the sensor during actuation of the miniature muscle:

- the conductive wires do not deform during actuation, their length and diameter stays constant;

- the pair of conductive wires stays parallel at any time during actuation;

- the conductive wires behaves as ideal conductors;

- the capacitance of the helical conductive wires can be approximated by the capacitance of a pair of straight parallel wires of same length. 
Therefore, the capacitance between both conductive wires of the sensor can be expressed as the capacitance between a pair of parallel wires:

$$
C=\frac{\pi \varepsilon l_{c}}{\ln \left(\frac{d}{r}+\sqrt{\frac{d^{2}}{4 r^{2}}-1}\right)}
$$

with $\varepsilon$, the permittivity of air, $l_{c}$, the length of the conductive wires, $d$, the distance between both parallel wires, and $r$, the radius of the conductive wires. It is assumed that $\varepsilon, l_{c}$ and $r$ stay constant during actuation of the artificial muscle (see hypotheses above). On the other hand, $d$ will vary.

Davis et al. defined the distance between two adjacent strands of a McKibben muscle as [22]:

$$
d_{a d j}=G \cdot \cos (\theta)
$$

where $G$ is the node separation distance of the braided mesh, and $\theta$, the braid angle (Fig. 3).

The node separation $G$ is given by [22]:

$$
G=\frac{\pi D_{0}}{N_{c}} \sin (\theta)
$$

where $N_{c}$ is the number of nodes in one circulation of the muscles circumference and $D_{0}$ is the theoretical muscle diameter when $\theta=90^{\circ}$. This diameter can be expressed as [23]:

$$
D_{0}=\frac{b}{n \pi}
$$

In (4), $b$ is the length of one strand and $n$ is the number of winding this strand forms around the muscle.

By combining (2), (3) and (4), the distance between two adjacent strands can therefore be expressed as:

$$
d_{a d j}=\frac{b}{n N_{c}} \sin (\theta) \cos (\theta)
$$
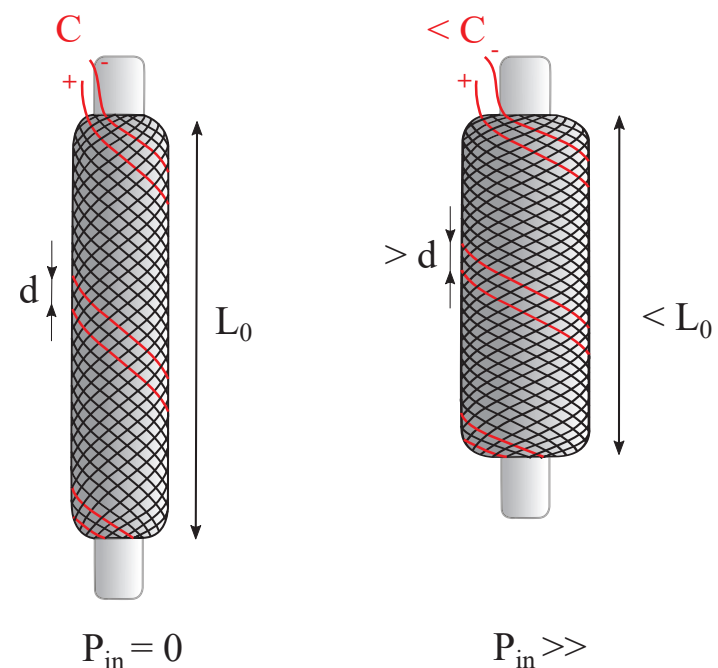

Fig. 2. Integrated capacitive sensor principle. Two strands of an original McKibben muscle are replaced by conductive wires (red wires). When pressurized, the muscle contracts, the distance between both wires $d$ increases and the capacitance between these two parallel wires $C$ decreases.

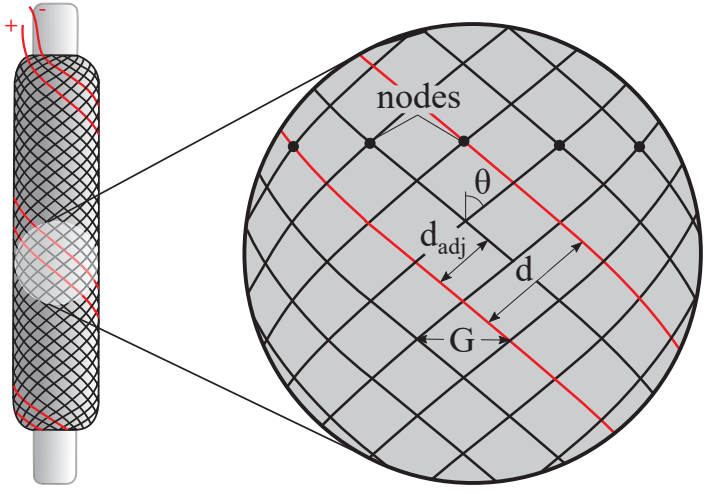

Fig. 3. Zoom on artificial muscle with integrated capacitance sensor with parameter definition for the theoretical sensor model [22].

The actuator length $L$ is given in function of $\theta$ by Chou et al. [6]:

$$
L=b \cdot \cos (\theta)
$$

By combining (5) and (6), $d_{a d j}$ can be expressed as a function of the muscle length:

$$
d_{a d j}=\frac{L}{n N_{c}} \sqrt{1-\left(\frac{L^{2}}{b^{2}}\right)}
$$

In (7), $n, N_{c}$ and $b$ are constants. Only the muscle length $L$ will vary during muscle actuation. The distance $d$ between the conductive wires is a multiple of $d_{a d j}$ depending on the number of strands between both conductive wires (Fig. 3). Therefore,

$$
d=\left(n_{s}+1\right) \cdot d_{a d j}
$$

where $n_{s}$ is the number of strands between the conductive wires. In the case presented in Fig. 3, $n_{s}=1$.

\section{INTEGRATED CAPACITANCE SENSOR: MECHANICAL AND ELECTRICAL PROPERTIES}

\section{A. Prototype manufacturing}

The manufacturing of the artificial muscle with integrated capacitance sensor is conducted as follows. First, one strand of the braided mesh is taken out and replaced by an enameled (insulated) copper wire of $0.1 \mathrm{~mm}$ diameter. The second copper wire is placed between two muscle strands at approximately equal distance of the first conductive wire all along the muscle length. Then, the braid with integrated sensor is slid on an inflatable silicone tube (Silastic RX50) with an external diameter $0.94 \mathrm{~mm}$ and an internal diameter $0.51 \mathrm{~mm}$. At one end, the muscle is sealed using a metal ferrule. At the other end, the bladder is connected to a non-inflatable tube, allowing a connection to the input air pressure. A ferrule is slid over this connection to protect it. The connection is strengthened by using a UV curing glue Dymax 204-CTH-F adhesive (Dymax, Wiesbaden, Germany). Figure 4a depicts the muscle with integrated capacitance sensor with all its components. This modified artificial muscle has a diameter of $2.6 \mathrm{~mm}$ and a length of $102 \mathrm{~mm}$ in deflected condition.

A second muscle with integrated sensor was manufactured 


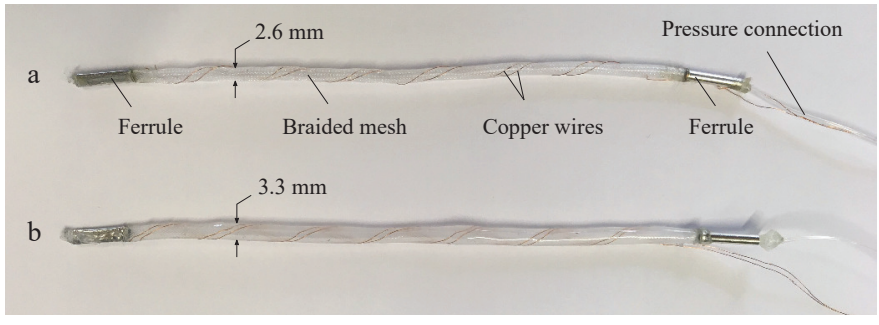

Fig. 4. Prototypes of an artificial muscle with integrated capacitance sensor; a) assembly of the muscle with integrated sensor. b) muscle with integrated sensor and with added silicone layer to stabilize the capacitance signal.

TABLE I

PROTOTYPES DESIGN CHARACTERISTICS

\begin{tabular}{cccc}
\hline Parameter & Value & Parameter & Value \\
\hline$\theta$ & $0.56 \mathrm{rad}$ & $r$ & $0.05 \mathrm{~mm}$ \\
$D_{0}$ & $2.6 \mathrm{~mm}$ & $l_{c}$ & $151 \mathrm{~mm}$ \\
$b$ & $151 \mathrm{~mm}$ & $n$ & 11.5 \\
$N_{c}$ & 22 & $n_{s}$ & 3 \\
\hline
\end{tabular}

with an additional silicone layer around the modified mesh. This layer helps to keep the copper wires in place during muscle actuation, preventing them from moving in a noncoordinated fashion during contraction/expansion of the muscle with integrated sensor. This therefore allows to stabilize the capacitance signal. In order to create that layer, the muscle with integrated sensor is immersed for a couple of seconds in an EcoFlex ${ }^{T M} 00-50$ bath. Figure $4 \mathrm{~b}$ shows the modified muscle with the added silicone layer. This muscle with integrated sensor and added silicone layer has a diameter of $3.3 \mathrm{~mm}$ and a length of $10 \mathrm{~mm}$ in deflected condition. The detailed characteristics of both modified muscles are gathered in Table I.

\section{B. Test bench}

In order to test the mechanical and electrical properties of both handmade muscles with integrated capacitance sensor (Fig. 4), a test bench was assembled. To assess the mechanical properties of the muscle with integrated capacitance sensor, three sensors are used, a load cell BCM 16687(S) $1 \mathrm{~kg}$ (BCM sensor, Antwerpen, Belgium), a pressure sensor Keller 21Y series (Keller, Winterthur, Switzerland) and a Baumer laser OADM 12I6460/S35A (Baumer, Frauenfeld, Switzerland). The sensors are interfaced with National Instruments modules NI9205 (Analog voltage input) and NI 9237 (Analog bridge input) plugged in a CompactRIO 9074 System. Sensors data was acquired using LabVIEW (National Instruments, Texas, USA). To assess the capacitance of the developed sensor, an electrical impedance spectrometer (Quadra, Eliko, Tallinn, Estonia) was used. The Quadra was interfaced with LabVIEW. Data was processed on Matlab (Mathworks, Massachusetts, USA). In order to regulate the pressure supplied to the muscle, a proportional pressure regulator VEAA from Festo (Festo, Esslingen am Neckar, Germany) was used in series with a
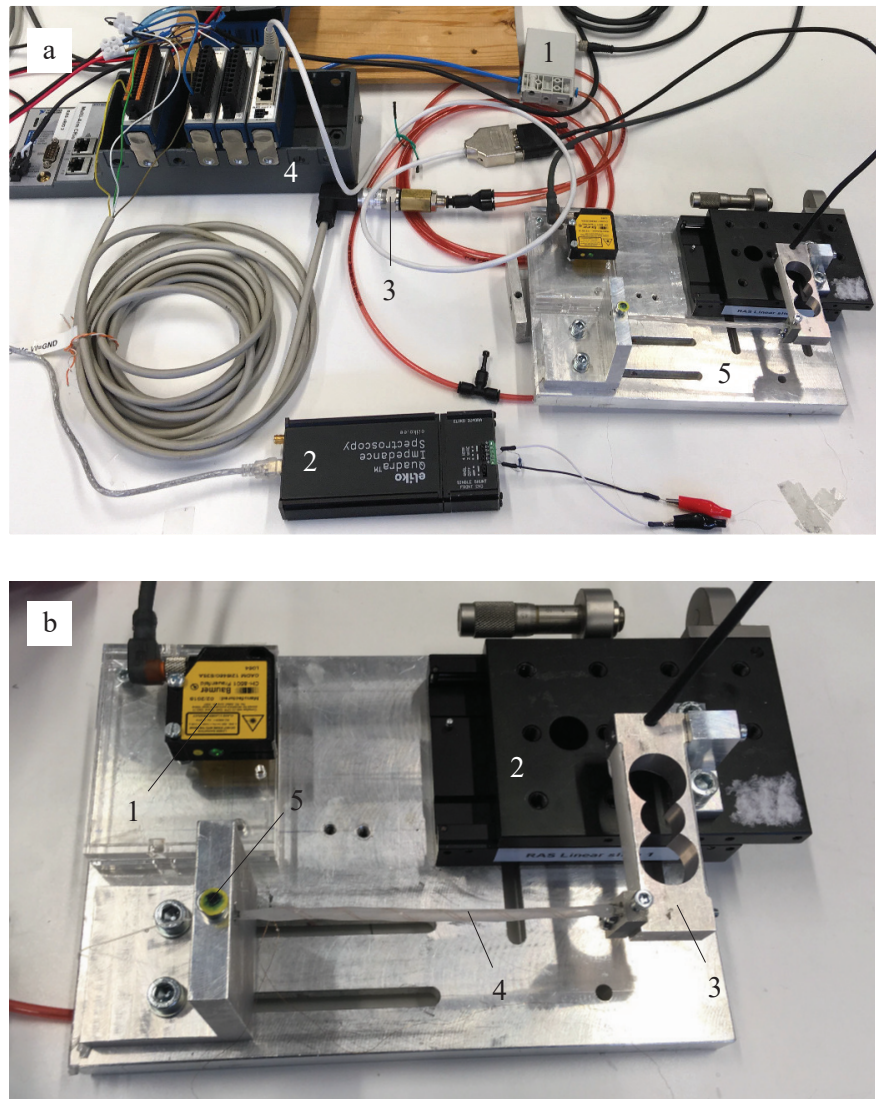

Fig. 5. Test bench to assess the mechanical and electrical properties of the artificial muscle with integrated capacitance sensor; a) Overview of the test bench: 1. proportional valve; 2 . impedance sensor; 3 . pressure sensor; 4. c-Rio and NI modules; 5. muscle test cell. b) Zoom on the test cell: 1. distance laser; 2. linear slide; 3 . load cell; 4. artificial muscle with integrated capacitance sensor; 5. fixed clamping to the frame.

compressor. The signal regulating the pressure was sent to the valve using a National Instruments module NI 9263 (Analog voltage output). The overall test bench is represented in Fig. $5 \mathrm{a}$. The test bench is composed of a test cell used to assess the mechanical and electrical properties of the artificial muscle with integrated sensor. Its composition is as follows. The load cell is fixed on a linear stage M-423 (Newport Corporation, California, USA). The modified muscle is clamped at one end to the frame, and at the other to the load cell. The laser is fixed to the frame in such a way that the laser beam hits the moving part of the slide. In order to sense the muscle contraction, the slide is let free and the position data is recorded by the laser. It is assumed that the friction involved in the slide is negligible and does not impede free motion along the muscle longitudinal direction upon contraction and relaxation. In a second round of experiments, where the force generated by the muscle with integrated sensor is measured, the slide is blocked, and data from the load cell is recorded. A zoom-view of the muscle test cell assembly is shown in Fig. 5b.

\section{Mechanical results}

The mechanical properties of both above mentioned muscle with integrated capacitance sensor were tested and compared 
to a handmade original McKibben muscle of the same diameter and length. The muscle length was assessed for the three muscles as a function of the input pressure over 10 cycles for each muscle. To do so, the linear slide was set loose and the pressure sensor and the laser were used to sense the input pressure and the muscle contraction distance respectively (Fig. 5b). The results of the muscle length tests are depicted in Fig. 6. The figure shows how the integrated capacitance sensor reduces the contraction range. The contraction was evaluated to be $20.1 \%$ for the muscle with integrated sensor and without silicone layer and $20.4 \%$ for the muscle with integrated sensor and with added silicone, whereas the original muscle has a contraction of $23.5 \%$. The addition of the silicone layer shows a reduction of the muscle hysteresis by half in comparison with the original muscle. The maximum hysteresis of the original muscle was evaluated to $77 \mathrm{kPa}$ with a mean

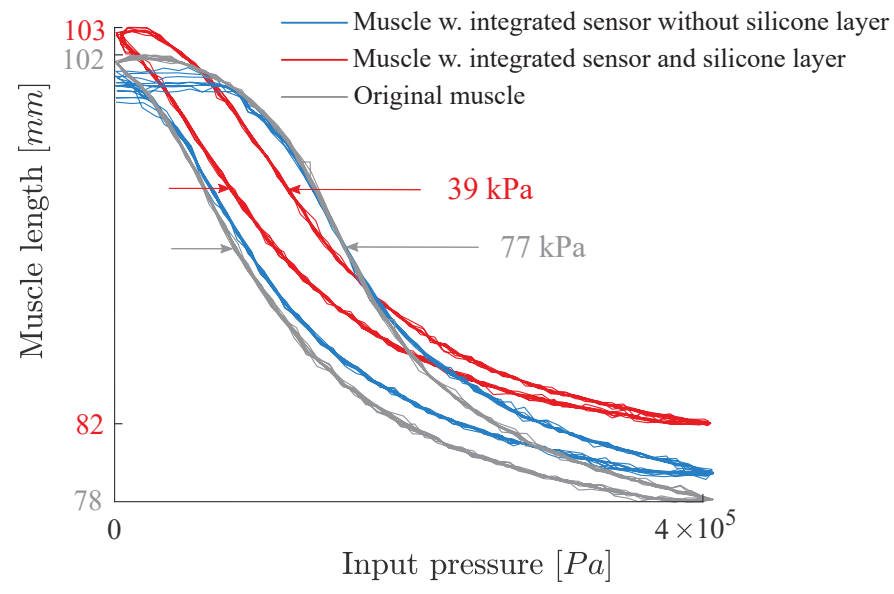

Fig. 6. Muscle contraction as a function of the input pressure (10 cycles for each muscle). The muscle with integrated sensor and silicone layer shows $20.4 \%$ of contraction, whereas the original muscle shows $23.5 \%$. The muscle with integrated sensor and silicone layer has a hysteresis half the size of the hysteresis of the original muscle.

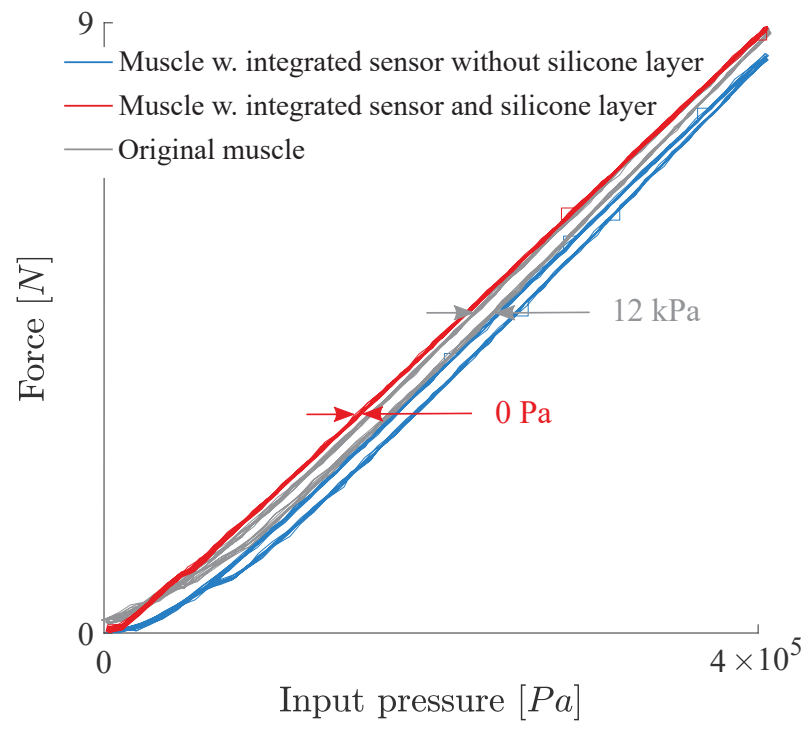

Fig. 7. Muscle output force as a function of the input pressure (10 cycles for each muscle). The muscle with integrated sensor and silicone layer provides the same range of force as the original muscle while canceling its hysteresis. hysteresis of $61 \mathrm{kPa}$ over the entire muscle length range. The maximum hysteresis of the muscle with integrated sensor and with silicone layer was found to be $39 \mathrm{kPa}$ with a mean hysteresis of $32 \mathrm{kPa}$ over the entire muscle length range.

The force generated by the three muscles was also evaluated as a function of the input pressure over 10 pressure cycles. To do so, the slide of the set-up was locked and the pressure and force sensor were used (Fig. 5b). The results of the force measurements are depicted in Fig. 7. It can be seen that the integration of a capacitance sensor does not affect the achievable force range. This is because the silicone layer of the muscle with integrated sensor prevents the braid angle to increase properly during contraction, limiting the muscle contraction. Since the generated force of an artificial muscle decreases as the muscle contracts [24], the generated force at maximum contraction of the muscle with silicone layer is higher than for an original muscle (which contracts fully) at same pressure. Note that the elastic modulus of the silicone layer ( $<0.5 \mathrm{MPa}$ at $200 \%$ of deformation) [25] is much smaller than for the muscle bladder (2.1MPa at $200 \%$ of deformation) [26]. Therefore, the extra energy needed to deform the silicone layer is not substantial and does not significantly lower the output force. From Fig. 7, it can also be seen that the use of a silicone layer around the modified muscle helps cancel the pressure-force hysteresis. The maximum and mean hysteresis of the original muscle were respectively evaluated to be $12 \mathrm{kPa}$ and $9 \mathrm{kPa}$.

\section{Electrical results}

The electrical properties of both prototypes, i.e. the muscles with integrated sensor and with and without silicone were tested. For this purpose, the Quadra impedance spectrometer was used. A resistor of $10 \mathrm{k} \Omega$ was placed in parallel with the muscle in order to avoid saturation of the spectrometer. The Quadra is capable of measuring 15 different frequencies simultaneously all at $1 \mathrm{kHz}$ sampling frequency. It can measure impedance magnitudes from $1 \mathrm{k} \Omega$ to $100 \mathrm{k} \Omega$ with $0.5 \%$ accuracy. The data was collected at a frequency of $179 \mathrm{kHz}$. This excitation frequency was found to be more sensitive than other frequencies. The obtained data was filtered using a median filter with a window size of 50 data points. Each muscle was subjected to 10 loading and unloading cycles. In Fig. 8, it can be seen that the silicone layer plays a significant role in reducing the noise and hysteresis over the cycles. The signal measured from the modified muscle without silicone layer is very noisy and presents hysteresis (Fig. 8a). This signal is difficult to use as a distance measurement due to its poor precision and large hysteresis. The signal measured on the muscle with integrated sensor and silicone layer is much more precise (Fig. 8b) due to the fact that the silicone layer prevents the copper wires from undesired motion. This signal (Fig. 8b) can therefore be used in the region where its sensitivity is large enough.

Fig. $8 \mathrm{~b}$ shows a parabolic relation between the muscle length and the capacitance. Therefore, in about $25 \%$ of the measurement range (from $89.2 \mathrm{~mm}$ to $84 \mathrm{~mm}$ in Fig. $8 \mathrm{~b}$ ), 


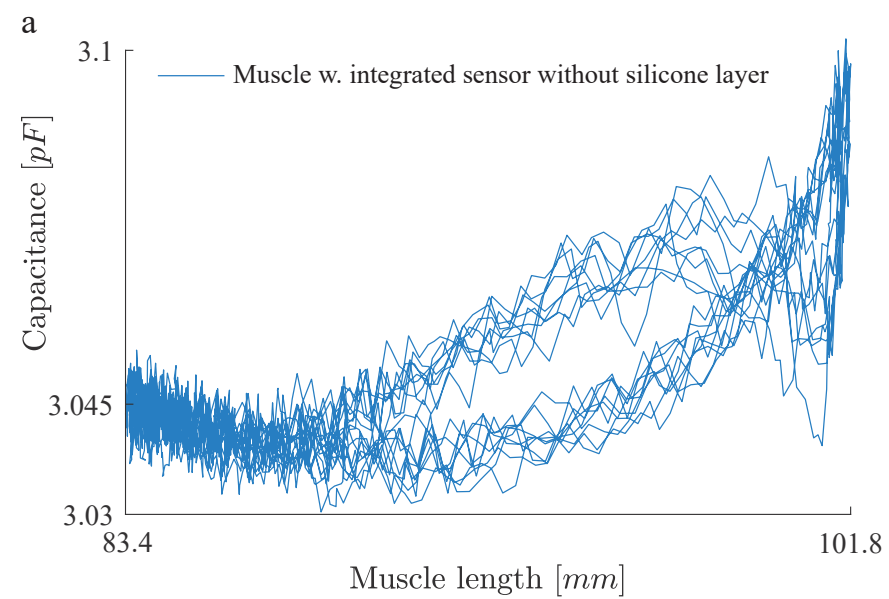

b Muscle w. integrated sensor and silicone layer (Experiments)

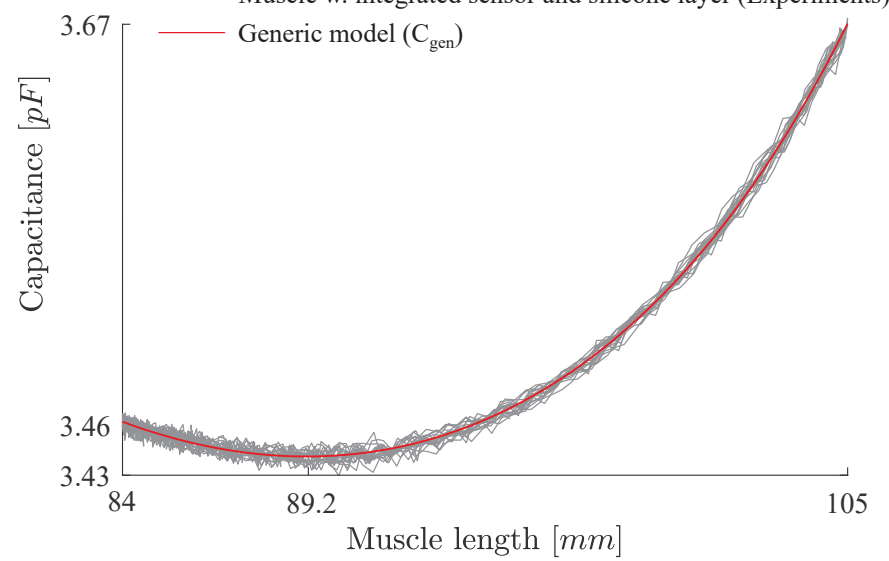

Fig. 8. Capacitance measurement upon muscle actuation (10 cycles for each muscle); a) capacitance measurement upon actuation of the muscle with integrated sensor and without silicone layer; b) capacitance measurement upon actuation of the muscle with integrated sensor and with silicone layer. Fitting of the generic model $C_{g e n}$, defined by (9), with $R^{2}=0.9971$.

it is not possible to distinguish the muscle length if the capacitance measurement is solely based on the sensor. For this reason, the remaining $75 \%$ of the muscle contraction range is used in this work. From Fig. 8b, it can also be noticed that the sensor sensitivity decreases as the muscle contracts. This phenomenon is described in more detail in Section IV-A.

In order to check if the theoretical sensor model established in section II is valid and matches the experiments, a generic model is formulated. The in-lab manufacturing process shows limited precision in placing the wires parallel to each other along the entire muscle length (see Fig. 3). Therefore, some deviations between the developed theoretical model and the experiments are expected. A more precise correspondence can be established by defining and fitting a generic model on the experimental curve. A generic capacitance model can be derived by combining Eqs. (1) - (8) by merging all muscle characteristic constants in four constants; $A, B, D$ and $E$ :

$$
C_{\text {gen }}=\frac{A}{\ln \left(B L \sqrt{1-D L^{2}}+\sqrt{E L^{2}\left|1-D L^{2}\right|-1}\right)}
$$

TABLE II

SENSOR THEORETICAL AND GENERIC MODEL PARAMETERS

\begin{tabular}{ccc}
\hline Parameter & Theoretical value & Generic model value \\
\hline$A$ & $9.65 \cdot 10^{-12}$ & $4.73 \cdot 10^{-12}$ \\
$B$ & 49.04 & 18.35 \\
$D$ & 65.11 & 62.65 \\
$E$ & 601.3 & 2212 \\
\hline
\end{tabular}

$C_{g e n}$ therefore represents the capacitance calculated on the basis of the theoretical model, preserving the capacitance dependence in the muscle length.

Figure $8 \mathrm{~b}$ shows how the generic model parameters $A, B, D$ and $E$ can be fit to the experimental data. The model parameters are gathered in Table II and compared to the theoretical values. The Curve Fitting toolbox of Matlab (MathWorks, Natick, USA) was used to find these generic model parameters. The R-square value of the model is evaluated to be 0.9971 , which indicates a strong fit.

\section{SEnsor CHARACTERIZATION}

In this section, a more in-depth study of the integrated capacitance sensor is developed. First, the sensitivity is defined and the influencing parameters are identified and discussed. Then, the sensor is characterized in terms of design repeatability, precision and drift. Finally, the behavior of the sensor is assessed under muscle loading.

\section{A. Sensitivity study}

In order to obtain a better understanding of the importance of the different sensor and muscle design parameters on the sensor behavior, a sensitivity analysis has been conducted. The purpose is to determine how the sensitivity can be maximized for a given muscle and sensor parameter variation. To do so, the derivative to $L$ of (1) is calculated:

$$
\frac{\partial C}{\partial L}=\frac{-\pi \varepsilon l_{c} \cdot \frac{\partial z}{\partial L}}{z \ln ^{2}(z)}
$$

with,

$$
z=\frac{d}{r}+\sqrt{\frac{d^{2}}{4 r^{2}}-1}
$$

where $d$ is defined by (8). The derivative of $z$ with respect to $L$ is calculated as:

$$
\frac{\partial z}{\partial L}=\frac{\partial d}{\partial L} \cdot\left(\frac{1}{r}+\frac{d}{4 r^{2} \sqrt{\frac{d^{2}}{4 r^{2}}-1}}\right)
$$

The derivative of $d$ with respect to $L$ can be expressed as:

$$
\frac{\partial d}{\partial L}=\frac{n_{s}+1}{n \cdot N_{c}} \cdot\left(\frac{b^{2}-2 L^{2}}{b^{2} \sqrt{1-\frac{L^{2}}{b^{2}}}}\right)
$$

By changing the value of some of the sensor and muscle design parameters, the sensitivity will vary. Fig. 9 illustrates 
these variations. Note that the values of Table I were used in Eq. 10-13 in order to plot the curves in Fig. 9. Typically, decreasing the number of strands between the conductive wires of the sensor, $n_{s}$, allows to increase the sensor sensitivity (Fig. 9a). However, manufacturing a sensor with a small $n_{s}$ is challenging since it is handmade. For the prototypes that were shown before, $n_{s}=3$ was chosen as a trade-off between sensitivity and manufacturability. Using a small braid angle for the muscle design, $\theta$, also allows to increase the sensitivity (Fig. 9b). However, decreasing the braid angle decreases the stroke of the artificial muscle [27]. Therefore, a compromise needs to be found between sensitivity and stroke. In Fig. 9b, it can be seen that the muscle braid angle also influences the zero sensitivity. At zero sensitivity, a difference in muscle length will not be perceived by the sensor. If the braid angle is small, the sensor can avoid a zero sensitivity. If the braid angle is larger, however, the sensor cannot longer be used over the
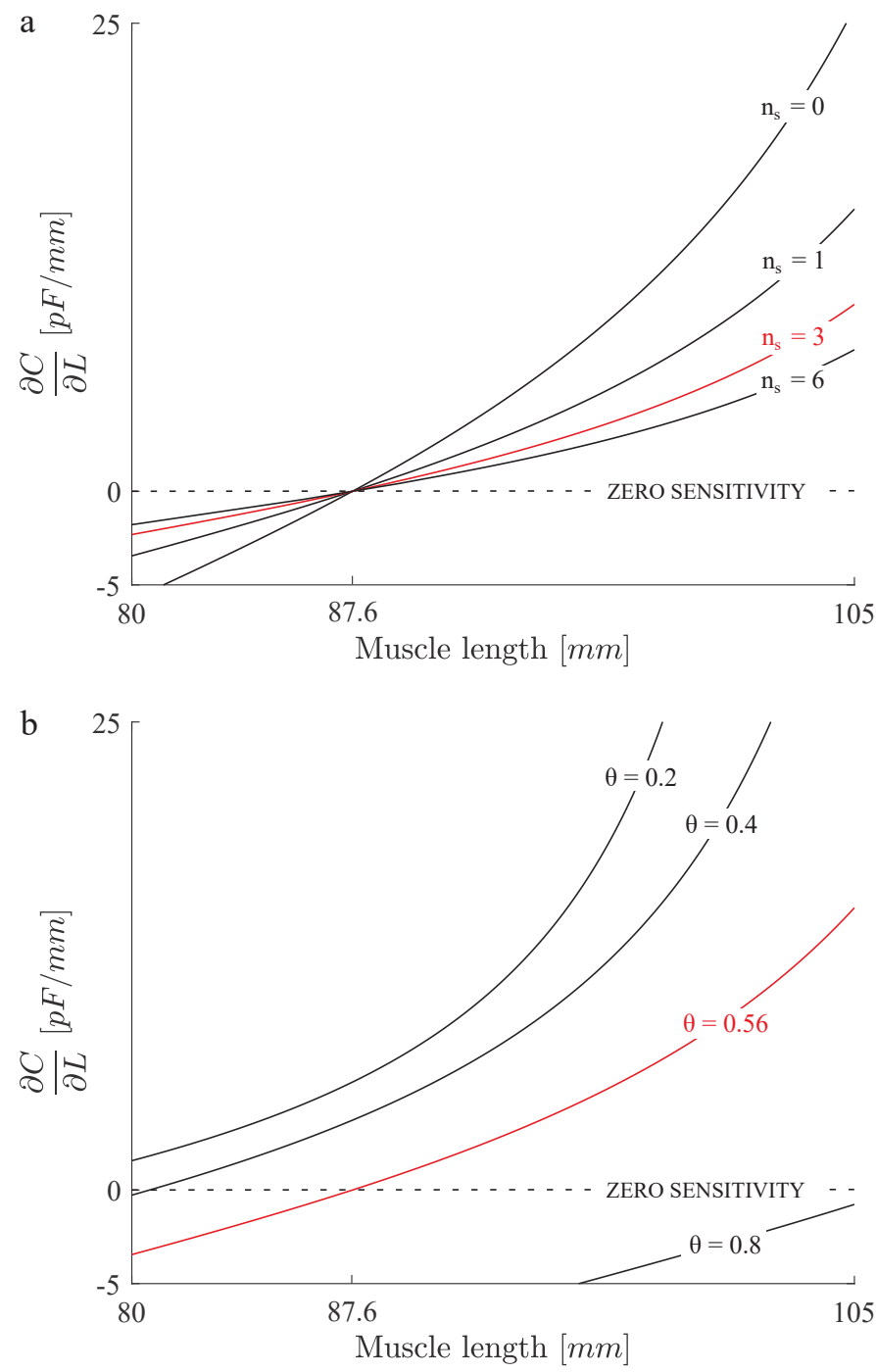

Fig. 9. Theoretical effect of model parameters on the sensor sensitivity (value used for the prototypes in red); a) effect of varying the number of strands between the sensor conductive wires; b) effect of varying the muscle braid angle $(\theta[\mathrm{rad}])$. full-muscle actuation range if the capacitive sensor is the only sensor used to predict the muscle length. For the prototypes that were shown before, a braid angle of $0.56 \mathrm{rad}$ was chosen.

The decrease in sensitivity when increasing the initial distance between the conductive wires of the sensor (i.e. when $n_{s}$ increases) could also be observed experimentally. In Fig. 10, three muscles with integrated capacitance sensor are tested with a different initial distance between the copper wires of their sensors. The wires are placed at $45^{\circ}, 90^{\circ}$ and $180^{\circ}$ from each other in the cross-section sketch of the muscle with integrated sensor (Fig. 10), which corresponds to a value of $n_{s}=3,5$ and 9 respectively. It can be seen that increasing the initial gap between the wires makes the capacitance signal upon actuation of the muscle flatter. For the wires placed at $180^{\circ}$ from each other, however, the sensitivity model (10) does not hold anymore since the distance between the wires equals the distance between each turn of the wire pair around the muscle $(d=e$ on Fig. 10). An extra capacitance therefore exists between the consecutive wire turns. From Fig. 10, however, this extra capacitive effect does not improve the sensor sensitivity.

The muscle and sensor parameters, typically $\theta$ and $n_{s}$, can be therefore be chosen in order to increase the sensitivity of the sensor in its usable range. By increasing the sensitivity, the relative effect of the sensor noise can become less prominent.

\section{B. Design variability}

In order to fully characterize the capacitive sensor, three identical muscles with integrated sensor were made. The sensor precision, generic model precision and drift are calculated for each of them, and the three sensors are

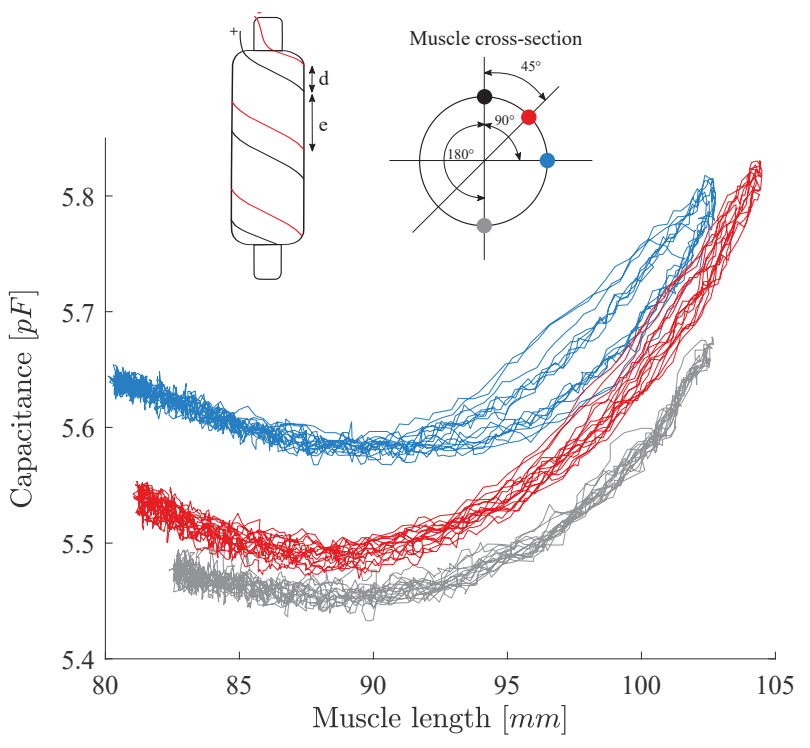

Fig. 10. Experimental effect of an increase of the distance between both conductive wires of the sensor. The black wire represents the reference wire. The wires are positioned at $180^{\circ}, 90^{\circ}$ and $45^{\circ}$ from each other in the crosssection plan of the muscle with integrated sensor. $d$ is the distance between both wire, $e$ is the distance between each turn of the wire pair around the muscle. 
compared to each other in order to evaluate the variability of the manufacturing process. These muscles with integrated sensor were designed using the parameters reported in Table I.

The total contraction range of these three muscles with integrated sensor is $22.1,21.8$ and $22.4 \mathrm{~mm}$ for the first, second and third muscle respectively. This difference in contraction range is due to the fact that all muscles, including the ones with integrated sensor, are hand-made and thus have slight variations in initial length that affect the total contraction range. The sensor, however, is only usable in the rising proportion of the capacitance curve (from 89.2 to $105 \mathrm{~mm}$ of muscle length in Fig. 8) if the sensor is used alone to determine the muscle length. This usable sensor range for the three muscles with integrated sensor are evaluated to be $79.69 \%, 81.51 \%$ and $77.31 \%$ of the total achievable contraction range for the first, second and third muscle respectively. Figure 11 shows the experimental results of the three muscles with integrated sensor over their usable range. Each muscle was tested over 20 loading and unloading cycles. The generic model (9) was also calculated for each muscle by using the Curve Fitting toolbox of Matlab to determine the model variables (i.e. $A, B, D$ and $E$ ). These model variables, as well as the sensor and generic model precision are gathered in Table III. From Fig. 11, it can be observed that the third prototype presents a more outspoken hysteresis behavior. We expect that is due to an imperfection during the placement of the silicone layer. Indeed, the silicone layer needs to fully cover the braided mesh and copper wires of the muscle. If that step it not correctly performed, the copper wires could come loose from the silicon and move freely during the muscle actuation, increasing hysteresis as visible in Fig. 8a. The capacitance sensor can therefore also be used to detect manufacturing defects. Overall, the manufacturing of the muscles with integrated sensor shows quite some variability (Fig. 11). A calibration of the sensor before its first use is therefore necessary. The generic model defined by (9) can be used for this calibration.

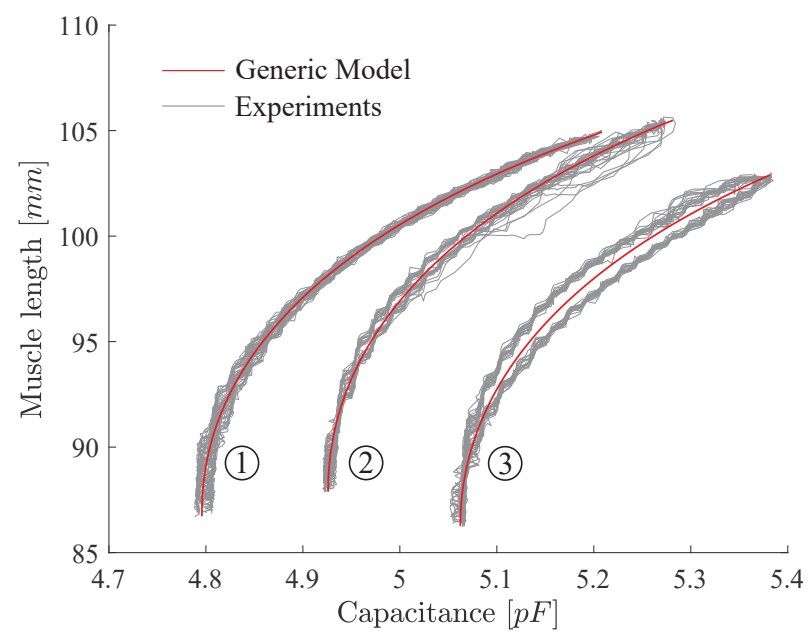

Fig. 11. Testing of three similar muscles with integrated sensor, and fitting of the generic model, defined by (9), for each of them (20 cycles for each muscle).
TABLE III

CHARACTERISTICS OF THREE MUSCLE WITH INTEGRATED SENSOR PROTOTYPES

\begin{tabular}{cccc}
\hline & Prototype 1 & Prototype 2 & Prototype 3 \\
\hline Model parameters & $A=8.295 \cdot 10^{-12}$ & $A=7.466 \cdot 10^{-12}$ & $A=8.934 \cdot 10^{-12}$ \\
& $B=3.645 \cdot 10^{-14}$ & $B=2.22 \cdot 10^{-14}$ & $B=2.439 \cdot 10^{-14}$ \\
& $D=64.83$ & $D=66.19$ & $D=67.48$ \\
$R^{2}$ model fit & $E=7788$ & $E=6224$ & $E=9486$ \\
Sensor precision [mm] & 0.9944 & 0.9978 & 0.9974 \\
Generic model precision [mm] & 0.699 & 0.784 & 1.089 \\
\hline
\end{tabular}

It is also important to mention that the prototypes on Fig. $8 \mathrm{~b}, 10$ and 11 were tested at different moments. It was found difficult to maintain the loose conductive wires going out from the muscles with integrated sensor the exact same way for each test session, as well as the exact same environment. Therefore, the absolute value of the measured capacitance may vary between each test. It is therefore recommended to calibrate the sensor once it has been integrated in its final system. An alternative method to the calibration for a more reliable placement and fabrication of the sensor would be the automation of the fabrication of the braided mesh with integrated sensor and the placement of the silicone layer. This automated process, however, would be very costly, especially for limited production.

The sensor precision was calculated by subtracting the maximum and minimum muscle length value for a given capacitance value. The average sensor precision over its usable range is estimated to be $0.699 \mathrm{~mm}$ and $0.784 \mathrm{~mm}$ for the first and second prototype respectively. Note that the average precision is largely determined by the poor sensitivity at low capacitance values. Due to its hysteresis, the average precision of the third prototype is a bit lower, with $1.089 \mathrm{~mm}$. The generic model precision of the three prototypes could also be calculated. This precision is based on the generic model (defined by (9)) calculated for each muscle and was evaluated to be $0.285 \mathrm{~mm}$, $0.196 \mathrm{~mm}$ and $0.462 \mathrm{~mm}$ for the first, second and third prototype respectively.

\section{Drift study}

The drift was also tested upon contraction of the muscle at $400 \mathrm{kPa}$ (maximum pressure the handmade McKibben muscle can endure) during 290s. By choosing the maximum pressure as a set point for the drift study, the worst-case scenario is considered. Indeed, it is at that pressure that the McKibben muscle is subject to the highest stress. A slight capacitance increase of $8 f F$ was recorded (Fig. 12a). This small increase is negligible since capacitance values in the order of $p F$ are measured by the sensor. This small increase is not due to a muscle elongation, since the recorded muscle length stays quasi-constant (Fig. 12b). Also, no drift is present when testing the Quadra impedance meter on a constant capacitance. Therefore, the conclusion can be drawn that the drift is 


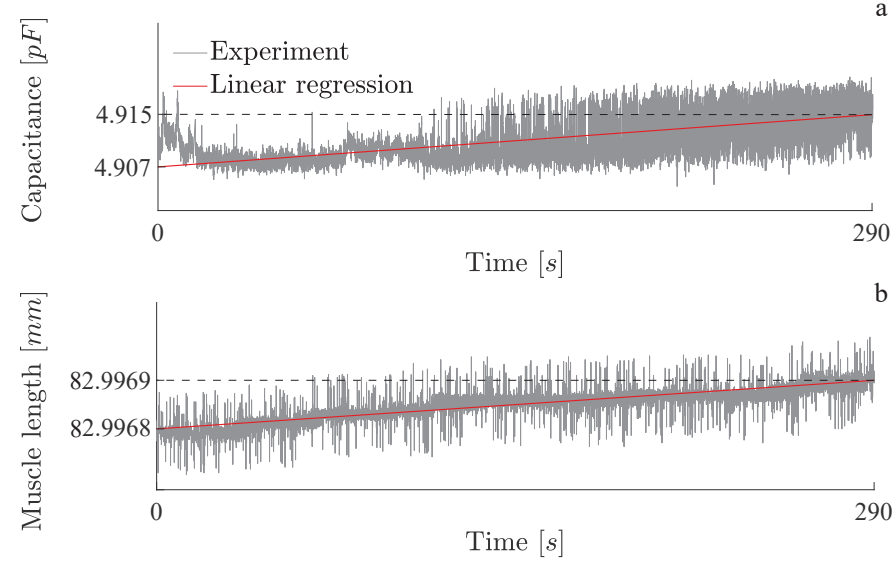

Fig. 12. Drift test upon contraction of the muscle with integrated sensor at $400 \mathrm{kPa}$ during $290 \mathrm{~s}$; a) capacitance measurement increasing with $0.008 \mathrm{pF}$; b) muscle length measurement increasing with $<1 \mu \mathrm{mm}$.

caused by the creep of the silicone layer resulting in a slight displacement of the copper wires.

\section{Force dependence}

The behavior of the sensor was also assessed under muscle external loading. In order to create the loading, the muscle length is fixed to its maximum, and the pressure gradually increases from 0 to $4 \cdot 10^{5} \mathrm{~Pa}$. Practically, the linear slide is blocked at the muscle maximum length (Fig. 5b) while the data from the load cell and Quadra are recorded. Figure 13 gives the results of the load test. The capacitance value decreases about $0.03 p F$ when the muscle output force increases as much as $8.5 N$. This variation represents approximately $8 \%$ of the sensor usable range of the three tested muscle with integrated sensor (Fig. 11). Felt et al. also reported this phenomenon [19]. In another study, Felt. et al proposed to model that phenomenon by considering the strain underwent by the conductive wires [20]. Although $8 \%$ error is rather small, further study on the control of the muscle with integrated sensor needs to be conducted in order to evaluate if such an error is admissible or if some methods need to be implemented to limit this effect.

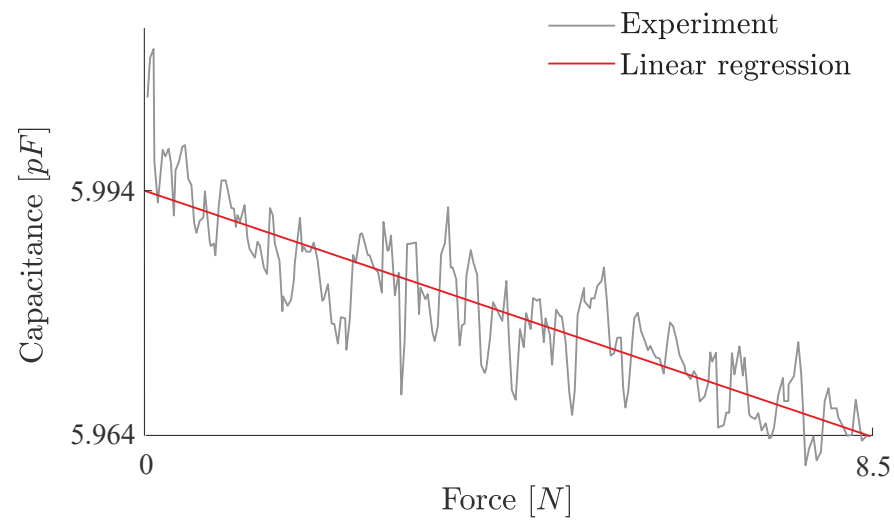

Fig. 13. Load test of the muscle with integrated sensor at constant muscle length. The measured capacitance decreases with $0.03 p F$ when the muscle output force reaches $8.5 \mathrm{~N}$.

\section{CONCLUSION}

The present study presented a novel easy producible integrated capacitance sensor for miniature artificial muscle actuators. The sensor is integrated in the actuator by replacing one strand of the muscle braided mesh with one thin conductive wire, as a second conductive wire is placed between two muscle strands. By measuring the capacitance between both wires with an impedance sensor during muscle actuation, the length of the muscle can be known. The sensor can only be used in a $80 \%$ contraction range of the muscle in average if the sensor is the only one used to determine the muscle length. For the $105 \mathrm{~mm}$ long and $3.3 \mathrm{~mm}$ external diameter muscles with integrated sensor that were developed, a sensor precision of $0.86 \mathrm{~mm}$ (i.e. $5 \%$ of the total muscle length range) was reached on average on the usable contraction range when the muscle was not loaded. The sensor can be calibrated based on an analytical model describing the capacitance effect between both conductive wires upon muscle actuation. The model precision was evaluated to be $0.31 \mathrm{~mm}$ (i.e. $1.8 \%$ of the total muscle length range) on the sensor usable range. Under muscle external loading, the capacitance of the sensor showed lower values with regard to an unloaded configuration. The loading can induce an error of approximately $8 \%$ on the total muscle length range. Further work will be dedicated to test the muscle with integrated sensor in different environments (in proximity of metallic components, of other measurement machines, ...) and study the effect on the capacitance measurement. The muscle with integrated sensor will also be tested in a closedloop control system and it will be assessed if the error of the muscle length estimation upon muscle loading has a substantial or minor effect on the muscle controllability.

\section{ACKNOWLEDGMENT}

This work was supported by a grant from the Belgian FWO [SB/1S98418N].

\section{REFERENCES}

[1] P. Berkelman, P. Cinquin, E. Boidard, J. Troccaz, C. Ltoublon, and J.-M. Ayoubi, "Design, control and testing of a novel compact laparoscopic endoscope manipulator," Proceedings of the Institution of Mechanical Engineers, Part I: Journal of Systems and Control Engineering, vol. 217, no. 4, pp. 329-341, Jun. 2003.

[2] M. De Volder, A. J. M. Moers, and D. Reynaerts, "Fabrication and control of miniature McKibben actuators," Sensors and Actuators A: Physical, vol. 166, no. 1, pp. 111-116, Mar. 2011.

[3] J. Legrand, M. Ourak, A. Javaux, C. Gruijthuijsen, M. A. Ahmad, B. V. Cleynenbreugel, T. Vercauteren, J. Deprest, S. Ourselin, and E. V. Poorten, "From a Disposable Ureteroscope to an Active Lightweight FetoscopeCharacterization and Usability Evaluation," IEEE Robotics and Automation Letters, vol. 3, no. 4, pp. 4359-4366, Oct. 2018.

[4] H. Li, K. Kawashima, K. Tadano, S. Ganguly, and S. Nakano, "Achieving Haptic Perception in Forceps Manipulator Using Pneumatic Artificial Muscle," IEEE/ASME Transactions on Mechatronics, vol. 18, no. 1, pp. 74-85, Feb. 2013

[5] A. J. M. Moers, M. F. L. De Volder, and D. Reynaerts, "Integrated high pressure microhydraulic actuation and control for surgical instruments," Biomedical Microdevices, vol. 14, no. 4, pp. 699-708, Aug. 2012.

[6] C.-P. Chou and B. Hannaford, "Measurement and modeling of McKibben pneumatic artificial muscles," IEEE Transactions on Robotics and Automation, vol. 12, no. 1, pp. 90-102, Feb. 1996.

[7] T. Vo-Minh, T. Tjahjowidodo, H. Ramon, and H. Van Brussel, "A new approach to modeling hysteresis in a pneumatic artificial muscle using the maxwell-slip model," IEEE/ASME Transactions on Mechatronics, vol. 16, no. 1, pp. 177-186, 2010. 
[8] S.-L. Xie, H.-T. Liu, J.-P. Mei, and G.-Y. Gu, "Modeling and compensation of asymmetric hysteresis for pneumatic artificial muscles with a modified generalized PrandtlIshlinskii model," Mechatronics, vol. 52, pp. 49-57, Jun. 2018.

[9] A. Capace, C. Cosentino, F. Amato, and A. Merola, "A multistate friction model for the compensation of the asymmetric hysteresis in the mechanical response of pneumatic artificial muscles," in Actuators, vol. 8, no. 2. Multidisciplinary Digital Publishing Institute, 2019, p. 49.

[10] T. Akagi, S. Dohta, Y. Kenmotsu, F. Zhao, and M. Yoneda, "Development of Smart Inner Diameter Sensor for Position Control of Mckibben Artificial Muscle," Procedia Engineering, vol. 41, pp. 105-112, Jan. 2012.

[11] S. Wakimoto, K. Suzumori, and T. Kanda, "Development of intelligent McKibben actuator," in 2005 IEEE/RSJ International Conference on Intelligent Robots and Systems, Aug. 2005, pp. 487-492.

[12] O. Erin, N. Pol, L. Valle, and n. Yong-Lae Park, "Design of a bioinspired pneumatic artificial muscle with self-contained sensing," Conference proceedings: ... Annual International Conference of the IEEE Engineering in Medicine and Biology Society. IEEE Engineering in Medicine and Biology Society. Annual Conference, vol. 2016, pp. 21152119, 2016.

[13] S. Kuriyama, M. Ding, Y. Kurita, T. Ogasawara, and J. Ueda, "Flexible sensor for Mckibben pneumatic actuator," in 2009 IEEE SENSORS, Oct. 2009 , pp. $520-525$.

[14] Y. Yamamoto, S. Wakimoto, and K. Suzumori, "Evaluation of electro conductive film and strain gage as displacement sensor for pneumatic artificial muscle," in 2011 IEEE International Conference on Robotics and Biomimetics, Dec. 2011, pp. 1206-1211.

[15] J. P. King, L. E. Valle, N. Pol, and Y. Park, "Design, modeling, and control of pneumatic artificial muscles with integrated soft sensing," in 2017 IEEE International Conference on Robotics and Automation (ICRA), May 2017, pp. 4985-4990.

[16] Y. Park and R. J. Wood, "Smart pneumatic artificial muscle actuator with embedded microfluidic sensing," in 2013 IEEE SENSORS, Nov. 2013, pp. 1-4.

[17] I. Hamamoto, T. Akagi, S. Dohta, and H. Matsushita, "Development of a Flexible Displacement Sensor Using Nylon String Coated with Carbon and Its Application for McKibben Actuator," in 2006 SICEICASE International Joint Conference, Oct. 2006, pp. 1943-1946.

[18] N. C. Goulbourne, S. Son, and J. W. Fox, "Self-sensing McKibben actuators using dielectric elastomer sensors," in Electroactive Polymer Actuators and Devices (EAPAD) 2007, vol. 6524. International Society for Optics and Photonics, Apr. 2007, p. 652414.

[19] W. Felt, K. Y. Chin, and C. D. Remy, "Contraction Sensing With Smart Braid McKibben Muscles," IEEE/ASME Transactions on Mechatronics, vol. 21, no. 3, pp. 1201-1209, Jun. 2016.

[20] W. Felt and C. D. Remy, "Smart braid: Air muscles that measure force and displacement," in 2014 IEEE/RSJ International Conference on Intelligent Robots and Systems, Sep. 2014, pp. 2821-2826.

[21] A. Devreker, "Design and control of continuum robots for minimally invasive surgical applications," Ph.D. dissertation, Katholieke Universiteit Leuven, 2017.

[22] S. Davis and D. G. Caldwell, "Braid Effects on Contractile Range and Friction Modeling in Pneumatic Muscle Actuators," The International Journal of Robotics Research, vol. 25, no. 4, pp. 359-369, Apr. 2006.

[23] H. F. Schulte, "The characteristics of the mckibben artificial muscle (1961) the application of external power in prosthetics and orthotics." National Academy of Sciences-National Research Council, Washington DC, 1961.

[24] F. Daerden and D. Lefeber, "Pneumatic artificial muscles: actuators for robotics and automation," European journal of mechanical and environmental engineering, vol. 47, no. 1, pp. 11-21, 2002.

[25] T. Ranzani, G. Gerboni, M. Cianchetti, and A. Menciassi, "A bioinspired soft manipulator for minimally invasive surgery," Bioinspiration \& biomimetics, vol. 10, no. 3, p. 035008, 2015.

[26] D. Corning. (2005) Silastic rx medical grade tubing. [Online]. Available: https://www.valcor.com/valcor-technical-datasheets/tubinginformation.pdf

[27] M. F. Gentry and N. M. Wereley, "Effects of braid angle on pneumatic artificial muscle actuator performance," in ASME 2008 Conference on Smart Materials, Adaptive Structures and Intelligent Systems. American Society of Mechanical Engineers, 2008, pp. 617-623. 